\title{
Automated Segmentation of Cerebral Vasculature with Aneurysms in 3DRA and TOF-MRA using Geodesic Active Regions: an Evaluation Study
}

\author{
Hrvoje Bogunović, José María Pozo, and María Cruz Villa-Uriol \\ Center for Computational Imaging 8 Simulation Technologies in Biomedicine (CISTIB) - \\ Universitat Pompeu Fabra (UPF) and Networking Biomedical Research Center on Bioengineering, \\ Biomaterials and Nanomedicine (CIBER-BBN), Barcelona, Spain \\ Charles B.L.M. Majoie and Rene van den Berg \\ Department of Radiology, Academic Medical Center, University of Amsterdam, Amsterdam, \\ The Netherlands \\ Hugo A.F. Gratama van Andel \\ Department of Biomedical Engineering \& Physics, Academic Medical Center, University of Amsterdam, Amsterdam, \\ The Netherlands \\ Juan M. Macho, Jordi Blasco, and Luis San Román \\ Department of Radiology, Hospital Clinic, Barcelona, Spain \\ Alejandro F. Frangi \\ Center for Computational Imaging 8 Simulation Technologies in Biomedicine (CISTIB) - \\ Universitat Pompeu Fabra (UPF) and Networking Biomedical Research Center on Bioengineering, \\ Biomaterials and Nanomedicine (CIBER-BBN) and \\ Institució Catalana de Recerca i Estudis Avançats (ICREA), Barcelona, Spain
}

(Dated: January 2011)

Purpose: To evaluate the suitability of an improved version of an automatic segmentation method based on geodesic active regions (GAR) for segmenting cerebral vasculature with aneurysms from 3D X-ray reconstruction angiography (3DRA) and time of flight magnetic resonance angiography (TOF-MRA) images available in the clinical routine.

Methods: Three aspects of the GAR method have been improved: execution time, robustness to variability in imaging protocols and robustness to variability in image spatial resolutions. The improved GAR was retrospectively evaluated on images from patients containing intracranial aneurysms in the area of the Circle of Willis and imaged with two modalities: 3DRA and TOF-MRA. Images were obtained from two clinical centers, each using different imaging equipment. Evaluation included qualitative and quantitative analyses of the segmentation results on 20 images from 10 patients. The gold standard was built from 660 cross-sections (33 per image) of vessels and aneurysms, manually measured by interventional neuroradiologists. GAR has also been compared to an interactive segmentation method: iso-intensity surface extraction (ISE). In addition, since patients had been imaged with the two modalities, we performed an inter-modality agreement analysis with respect to both the manual measurements and each of the two segmentation methods.

Results: Both GAR and ISE differed from the gold standard within acceptable limits compared to the imaging resolution. GAR (ISE, respectively) had an average accuracy of $0.20(0.24) \mathrm{mm}$ for $3 \mathrm{DRA}$ and 0.27 (0.30) $\mathrm{mm}$ for TOF-MRA, and had a repeatability of $0.05(0.20) \mathrm{mm}$. Compared to ISE, GAR had a lower qualitative error in the vessel region and a lower quantitative error in the aneurysm region. The repeatability of GAR was superior to manual measurements and ISE. The inter-modality agreement was similar between GAR and the manual measurements.

Conclusions: The improved GAR method outperformed ISE qualitatively as well as quantitatively and is suitable for segmenting 3DRA and TOF-MRA images from clinical routine.

Keywords: cerebral angiography; cerebral aneurysms; vessel segmentation; geodesic active regions; quantitative evaluation

\section{INTRODUCTION}

Cerebral aneurysm is a vascular pathology that tends to appear near bifurcations of arteries in the Circle of Willis. Although aneurysm prevalence is relatively low, estimated to be between $1 \%-5 \%^{1}$, aneurysm rupture causes sub-arachnoid haemorrhage $(\mathrm{SAH})$ having a high fatality rate (between $40 \%$ and $60 \%)^{2}$. Traditionally, for aneurysm detection, catheter 2D digital subtraction angiography (DSA) was considered as gold standard ${ }^{2}$ but lately it is being supplemented or replaced by $3 \mathrm{D}$ ro- tational X-ray angiography (3DRA $)^{3}$. Due to its superior spatial and contrast resolution, 3DRA is normally also used for treatment planning (surgical clipping or endovascular intervention). However, for diagnosis and follow ups, preference is given to noninvasive computed tomography angiography (CTA) and magnetic resonance angiography (MRA): contrast enhanced CE-MRA and time-of-flight TOF-MRA ${ }^{4}$.

Ruptured aneurysms are almost always treated. However, in the case of unruptured aneurysms, the indication for preventive treatment is not straightforward and the 
risk of treatment has to be carefully balanced against the risk of rupture. Computational modeling is increasingly used to characterize the aneurysms in order to find suitable predictors of risk of rupture ${ }^{5}$. Most notably, aneurysm shape characterization ${ }^{6,7}$ and analysis of its hemodynamic features using computational fluid dynamics $(\mathrm{CFD})^{8,9}$ are becoming increasingly important. These results are strongly determined by the modeled geometry of the aneurysms and surrounding vessels. Therefore, vascular segmentation from radiographic images is a key methodology in the computational analysis of the vasculature.

The segmentation of cerebral vasculature with aneurysms is a difficult task often due to their complex geometry as well as limited image contrast and spatial resolution, which are critical factors compared with the size of these vascular segments. Throughout their management cycle, patients undergo a variety of imaging examinations with various modalities. In addition, patients with ruptured aneurysms have 3DRA and CTA scans while patients with unruptured ones are preferably followed only with MRA. Thus, it is desirable to apply a cerebrovascular segmentation method able to cope coherently with different imaging modalities so that computational results are comparable. Furthermore, it is important that the applied segmentation method is completely automatic. This has a two-fold advantage. First, the segmentation method should be objective (operatorindependent) and highly repeatable to minimize variability propagation to other steps of the analysis pipeline. Second, requiring manual interaction from the operator increases the processing time and needs the allocation of human resources.

To the best of our knowledge no effective solution is currently available for the automatic and multimodal segmentation of cerebral vasculature with aneurysms. Recently, Manniesing et al. ${ }^{10}$ reported a successful feasibility study for the automatic segmentation of CTA images but their evaluation did not cover aneurysms, thus it is not clear how their results would extrapolate to diseased parts of the vasculature. Hernandez et al. ${ }^{11}$ proposed a method based on a geometric deformable model called geodesic active regions (GAR) and presented promising results for automatic segmentation of cerebral aneurysm from different modalities (3DRA and CTA). However, limiting factors for its application in clinical routine was its lack of robustness to the variation in imaging protocols and its elevated computational costs.

The purpose of this study was to introduce improvements into the GAR method and to evaluate its potential and limitations in segmenting cerebral vasculature with aneurysms from 3DRA and TOF-MRA images available in the clinical routine, including different clinical centers and imaging equipment. The evaluation was performed retrospectively on images of patients having cerebral aneurysms. The segmentation results were compared to the gold standard obtained from manual measurements performed by interventional neuroradiol- ogists. To analyze the segmentation consistency across the two imaging modalities, patients having both 3DRA and TOF-MRA examinations were chosen.

\section{MATERIALS AND METHODS}

\section{A. Patient Selection}

Ten patients (3 male, 7 female; age range: $35-70$; mean age: 57 years) with 16 cerebral aneurysms scanned with 3DRA and TOF-MRA within a short period (1 week) were retrospectively selected from two different clinical centers ( 7 from Academic Medical Center (AMC), The Netherlands and 3 from Hospital Clinic i Provincial de Barcelona (HCPB), Spain). Each center used different imaging equipment. Anonymized imaging data sets were used and the institutional review board approved the study, which had no influence on patient management. From the database of suitable candidates, patients were selected on the basis of the location of aneurysm to ensure that location variability followed their natural probabilities of occurrence as reported $\mathrm{in}^{1}$. For patients having multiple aneurysms only those present in both 3DRA and MRA images were eligible. Eventually, one aneurysm per patient was singled out. The different locations were: internal carotid artery (3), posterior communicating artery (2), anterior communicating artery (1), middle cerebral artery (3) and basilar tip (1). Aneurysms varied in size: small aneurysm of 3-5 mm (5), medium aneurysm of 8-9 $\mathrm{mm}$ (4) and a large aneurysm of $19 \mathrm{~mm}$ (1).

\section{B. Scanning Protocols}

3DRA images from AMC (HCPB, respectively) were acquired with a single-plane angiographic unit: Integris Allura Neuro; Philips Healthcare, Best, The Netherlands (AXIOM Artis; Siemens Medical Solutions, Erlangen, Germany). Non-ionic contrast agent: $320 \mathrm{mgI} / \mathrm{mL}$ iodixanol, Visipaque (240 mgI/mL iohexol, Omnipaque), GE Healthcare, Cork, Ireland, was injected through a $6 \mathrm{~F}$ catheter positioned in an internal carotid artery or vertebral artery. 100 images (120 images) were acquired during a $240^{\circ}\left(180^{\circ}\right)$ rotational run in 8 seconds with 15 to $21 \mathrm{ml}$ contrast agent at $3 \mathrm{ml} / \mathrm{s}$. On a dedicated workstation, 3D images were reconstructed with a $256^{3}$ matrix with a reconstructed voxel size of $0.19 \times 0.19 \times 0.19 \mathrm{~mm}$ $(0.39 \times 0.39 \times 0.39 \mathrm{~mm})$.

$\mathrm{MR}$ imaging examinations in $\mathrm{AMC}(H C P B$, respectively) were performed on a 3.0-Tesla system: Intera R10; Philips Healthcare, Best, The Netherlands (TrioTim; Siemens Medical Solutions, Erlangen, Germany). The protocol included 3D TOF-MRA with multiple overlapping thin slab acquisition MOTSA (with single slab acquisition). The parameters were as follows: TR/TE, $21 / 4 \mathrm{~ms}(23 / 4 \mathrm{~ms})$; flip angle, $20^{\circ}\left(15^{\circ}\right) ; 512 \times 326(640 \times$ 288) acquisition matrix, reconstructed to $1024 \times 1024$ 
$(640 \times 480) ; \mathrm{FOV}, 200 \times 170 \mathrm{~mm}(200 \times 150 \mathrm{~mm}), 85 \%$ $(75 \%)$ rectangular FOV; $1.0 \mathrm{~mm}$ thick sections interpolated to $0.5 \mathrm{~mm}$ (0.5 $\mathrm{mm}$ thick sections); 220 sections (160 sections) acquired, resulting in a coverage area of $110 \mathrm{~mm}$ $(80 \mathrm{~mm})$. The measured voxel size was $0.39 \times 0.61 \times 1$ $\mathrm{mm}(0.31 \times 0.52 \times 0.5 \mathrm{~mm})$, and the reconstructed voxel size was $0.2 \times 0.2 \times 0.5 \mathrm{~mm}(0.31 \times 0.31 \times 0.5 \mathrm{~mm})$. The scanning time was reduced with sensitivity encoding SENSE parallel imaging (generalized autocalibrating partially parallel acquisition GRAPPA), with a reduction factor of 1.5 (2), which resulted in an acquisition time of 7 min (4.5 $\mathrm{min})$.

\section{Segmentation Method}

The workflow of the automatic vascular segmentation method GAR is shown in Fig. 1. The core of the methodology was originally presented in $^{11}$, and in $^{12}$ the image intensity standardization (IIS) filter was introduced. In this paper, we improve some of the components of the workflow, but we first summarize the methodology that has remained unchanged.

The method is based on the geometric deformable model within the level set framework ${ }^{13}$. The internal force of the deformable model is defined as the local curvature of the evolving surface, while the external one combines region-based descriptors with gradient ones to drive the evolution of the model towards the vascular boundaries. The equation driving the evolution of the surface is expressed as:

$$
\frac{\partial}{\partial t} \Phi+\zeta\left(k_{\text {out }}-k_{\text {in }}\right)|\nabla \Phi|-\eta\left(\varepsilon g K_{m}|\nabla \Phi|+\nabla g \cdot \nabla \Phi\right)=0
$$

where $\Phi$ is an implicit function whose zero level set at any time $t$ of the evolution represents the vascular surface. The gradient descriptor $g$ is inversely proportional to the gradient magnitude of the image and helps stopping the evolution at the vessel boundary. The descriptor $K_{m}$ is the minimal curvature of the level set surface at each point, which favors the smoothness of the surface. The region descriptors $k_{\text {in }}$ and $k_{\text {out }}$ are defined by

$$
k_{\text {in }}(\mathbf{x})=-\log \left(P_{\text {in }}(\mathbf{x})\right) ; k_{\text {out }}(\mathbf{x})=-\log \left(P_{\text {out }}(\mathbf{x})\right)
$$

where $P_{\text {in }}$ and $P_{\text {out }}$ are the probabilities that the voxel $\mathbf{x}$ belongs to inner and outer regions with respect to the vascular lumen. The constant parameters $\varepsilon, \zeta$ and $\eta$ control the influence of the curvature, region-based and gradient-based descriptors, respectively, and were fixed as published in ${ }^{11}$.

In general, the GAR workflow is divided into two main phases:

Off-line training phase: Performed only once for each imaging modality. Images forming the training set are passed through IIS filter and interactively labeled into three regions: vessel, background and undecided region, which normally corresponds to partial volume voxels. Then, the feature vectors are calculated at training points randomly selected from vessel and background regions. Finally, the fuzzy classifier is trained in a supervised fashion on the set of feature vectors. The output is a set of parameters of the classifier used to compute the probabilities $P_{\text {in }}$ and $P_{\text {out }}$ during the segmentation phase.

Segmentation phase: The image being segmented is first passed through the IIS filter. At each image voxel, the feature vector is calculated and the probability of belonging to a particular region is estimated with the previously trained classifier. In parallel, the gradient magnitude is computed. Following Eq. 1, the level sets associated with $\Phi$ evolve toward a local minimum of the energy functional trying to maximize the probability for the vessel region inside the zero level set and the probability for the background region outside, taking also into account the curvature and the gradient information at the boundaries. The zero level set at the steady solution corresponds to the segmented vessel lumen surface. The resulting surface is extracted as a $3 \mathrm{D}$ triangular mesh with sub-voxel precision.

Such methodology is specially suitable for vessel segmentation from different modalities, since for each one the region descriptors are learned from an appropriate training set of images.

In this paper, we have introduced the following three improvements to the components of the workflow: The classifier has been replaced, the computation of the feature vector has been modified and the IIS technique has been adapted to handle large deformations.

a. Multiple valued neuron classifier. The originally proposed probability estimation was performed using $\mathrm{k}$ nearest neighbors $(\mathrm{k}-\mathrm{NN})$ algorithm on the training set. However k-NN is not convenient for fuzzy classification and is highly computationally demanding since during the classification it repeatedly searches for the nearest neighbors within the stored training feature vectors. Thus, we replaced it with the multiple valued neuron $(\mathrm{MVN})^{14}$. This improves the classification speed by approximately 8 times since most of the computational cost is in the training phase, which is performed only once. Furthermore, larger sets of points ( $1 M$ as opposed to $20 k$ used with k-NN) were used for training as their size does not influence the classification speed of MVN. Using more points allowed building a training set with larger number of images thus achieving better classifier generalization.

b. Voxel-based scale space. Features forming the feature vector are computed on multiple scales. To handle a large variety of possible image resolutions, the range of scales used for feature calculations is now based on image voxel spacing instead of being predefined and fixed. More details on how features are calculated are given in Appendix. 


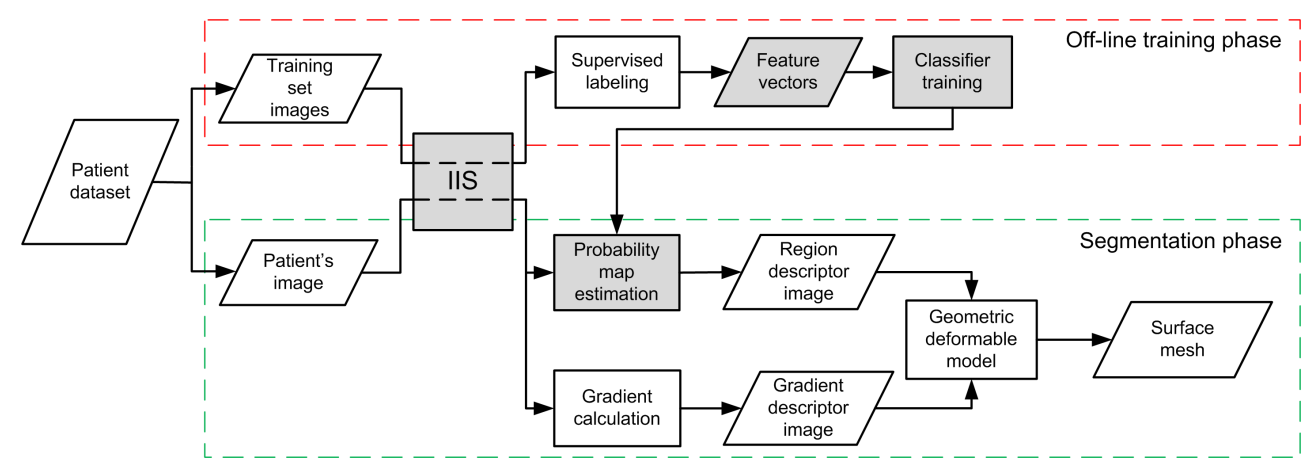

FIG. 1. Workflow of the automatic vascular segmentation method GAR. New or improved components are highlighted in gray.

c. Image intensity standardization (IIS). To get good estimates of the probability map, the images composing the training set should describe well the image to be segmented. In MRA and 3DRA, the intensities do not have a direct physical interpretation and the intensity range for the same tissues can largely vary between images. The IIS ${ }^{15}$ ensures that the intensity ranges corresponding to the same tissues are similar by establishing a correspondence between histograms taken from generic images and a histogram taken as a reference. The image histogram is aligned to the reference using a $1 \mathrm{D}$ non-linear registration technique. The generated intensity transformation is applied to the image volume, standardizing the intensity ranges. This enables the use of the same training set for any image of the same modality $^{12}$. In this study, we improved the registration technique to make it robust to large deformations and applicable to both 3DRA and MRA images. Normalized cross-correlation is used as a similarity metric and the deformation is parameterized by cubic B-spline. Limited memory Broyden Fletcher Goldfarb Shannon with simple bounds (LBFGSB) algorithm ${ }^{16}$ is used as optimizer. The registration is first initialized with a transformation composed of two control points aligning the histogram peaks and the largest intensity values. The registration is then performed following a multiscale approach: in three chained iterations, progressively incrementing the number of control points $(4,5$ and 6$)$, resetting their position equidistantly at the beginning of each iteration. The range of movement is limited to half the inter-control points distance ${ }^{17}$. This results in a diffeomorphic transformation since it is smooth and avoids folding. Although there is a constraint on the overall deformation, allowing further stretching or compression was not found necessary and it prevents instabilities. The IIS was able to align the histograms in both the 3DRA and MRA images of the patients (Fig. 2).

\section{Segmentation Evaluation Method}

One aneurysm and three surrounding vessels of clinical interest, both proximal and distal from the aneurysm

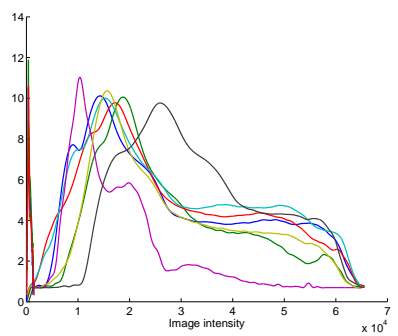

(a) 3DRA, before IIS

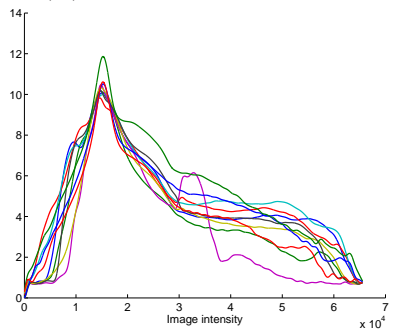

(c) 3DRA, after IIS

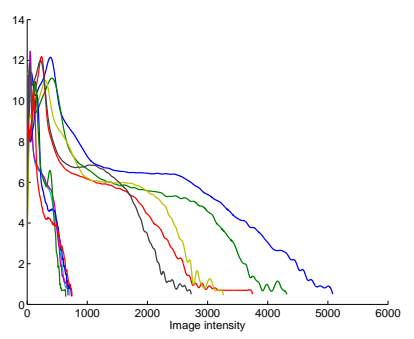

(b) MRA, before IIS

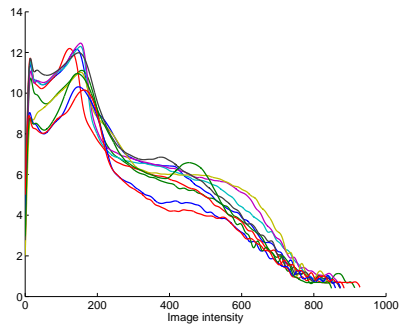

(d) MRA, after IIS
FIG. 2. Image histograms of the 10 patients before $(\mathrm{a}, \mathrm{b})$ and after (c, d) image intensity standardization of 3DRA (a, c) and MRA (b, d) images. The logarithm of histogram values is displayed.

(blood flow inlets and outlets) were selected per patient. The selected vessels and aneurysm had to be visible in both 3DRA and MRA scans of the patient. On every image, for each selected vessel 10 cutting planes were automatically positioned $1 \mathrm{~mm}$ apart and orthogonal to the vessel centerline. Three additional cutting planes were manually positioned on the aneurysm, one cutting the neck and the other two passing through the center of the neck and the maximum aneurysm depth. This yields 33 cutting planes per image (660 in total). Once defined, the cutting planes were kept fixed throughout the evaluation study. The positions of the cutting planes were chosen to representatively sample the geometry of interest around aneurysm. Having a good segmentation of that region is important for subsequent computational modeling approaches.

The cutting planes applied to 3D intensity image produce $2 \mathrm{D}$ images with the vessel cross-section, where the 
manual measurements were performed. The same cutting planes applied to segmented surface mesh produce $2 \mathrm{D}$ contours, on which the same measurements were automatically extracted. Since the cutting planes were fixed, all the measurements were taken on the same crosssections. Two measurements per cross-section were performed. Their definition together with the positioning of the cutting planes and examples of measurements of the cross-sections are shown in Fig. 3.

The shape of the vessel and aneurysm neck crosssection was assumed to be round but not necessarily circular. We proposed to describe the shape by the two orthogonal widths, which capture its major and minor axes and reduce the ambiguity when performing the manual measurements. Measurements of the dome are relative to the neck plane and its center. For each aneurysm neck cross-section, its center was computed. Thus, all the parameters of the neck were fixed and did not introduce variability into the measurements of the dome.

To build the gold standard, manual measurements performed by 5 interventional neuroradiologists from two clinical centers in 2 sessions were averaged. The measuring guidelines were easily followed by the clinicians performing the manual measurements. We also compared the GAR to another segmentation method: interactive contouring based on iso-intensity surface extraction (ISE). The iso-intensity surface is the interface between the regions of the image $I: I \geq c$ (the inside) and $I<c$ (the outside), where $c$ is a constant intensity value. The surface is obtained by marching cubes $\operatorname{method}^{18}$ where the points at which the iso-intensity surface intersects the edges of the voxel cube are obtained by linear interpolation, producing a triangular mesh with sub-voxel accuracy. Four expert users, with more than 3 years of angiographic experience, performed ISE segmentation in 2 independent sessions.

In order to evaluate the inter-modality agreement, a correspondence between cutting planes in both imaging modalities was established for each patient. Cutting planes on vessels were manually aligned, starting the cutting sequence at the same anatomical position in both imaging modalities. To establish corresponding aneurysm cutting planes, the 3DRA and MRA segmented surface models were first rigidly registered. Then the cutting planes that were manually positioned in 3DRA were transferred to their corresponding locations in MRA.

The rigid registration was performed in a supervised fashion. First, corresponding pairs of points on the two meshes were identified and an initial transformation was applied to approximately align them. Next, the registration was refined using the Iterative Closest Point algorithm ${ }^{19}$. This was repeated until the two meshes were adequately registered after visual inspection (Fig. 4). To evaluate the repeatability of such procedure, the registration was performed three times for each pair of meshes.

\section{Qualitative analysis}

The segmentation results were qualitatively evaluated by visual inspection of the cross-sections obtained from the segmentation results. The cutting planes where the segmented contours were missing, unrealistically deformed or merged with neighboring vessels, were classified as erroneous. Their occurrence rate and the effects causing them were analyzed. However, they were excluded from the subsequent quantitative analysis, since they would otherwise introduce large errors, which are not representative of the segmentation performance in denoting the actual border of the vessel lumen.

\section{Quantitative analysis}

For the quantitative analysis, three main factors, as proposed by ${ }^{20}$, were considered: repeatability, accuracy and efficiency. In addition, we also evaluated the global correctness by region overlap with manual segmentations and the inter-modality reproducibility.

Repeatability: The main variability of GAR results is introduced by the differences between the images used as a training set for the classifier. This variability was estimated from results obtained with different training sets. In addition, we evaluated the influence that the number of images in the training set has on the variability. The variability of manual measurements and ISE results is introduced by the observer, thus we estimated their inter- and intra-observer variability.

Accuracy: For GAR and ISE methods it was estimated in two complementary ways. First, as an average absolute difference from the gold standard. Second, as limits of agreement with the gold standard, which represent the bias and the $95 \%$ likely range for the differences. From this, a variability index was introduced where deviations from the gold standard are put into perspective by comparing them to the variability of manual measurements.

Region overlap: To complement the accuracy measurements, the segmentation results were converted from mesh to voxel representations and compared to manual segmentations performed on the geometry of interest by two experienced observers. Although less sensitive to small, sub-voxel variations, this more global metric can detect serious missegmentations of the vessel region. Two region overlap measures, as used in the recent works ${ }^{21,22}$, were calculated: the Dice $^{23}$ and the conformity ${ }^{24}$ scores.

Efficiency: Estimated as average time spent in segmenting an image. For automatic GAR method it was the computer execution time while for interactive 


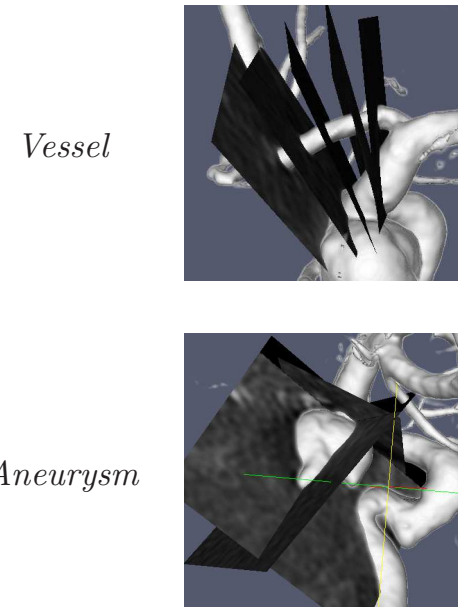

(a)
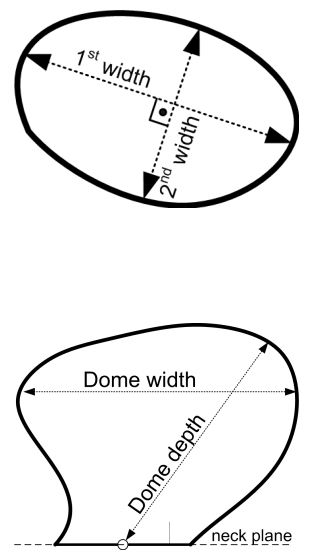

(b)
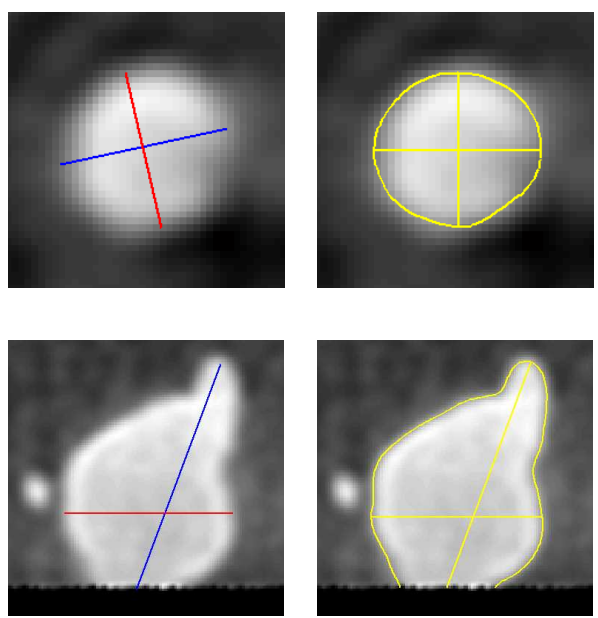

(c)

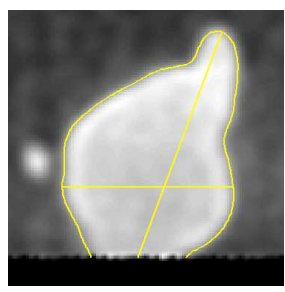

$(d)$

FIG. 3. (a) Positioning of cutting planes. (b) For measuring vessels and aneurysm neck the two measuring segments need to be orthogonal to each other ( $1^{\text {st }}$ width is chosen first) and located where the width is maximum. Dome depth is the distance from the neck center to the farthest point on the dome, while dome width is the length of the longest chord of the dome parallel to the neck plane. (c) Example of manual measurements. (d) Example of automatic measurements.

ISE, was the time spent by the observer deciding on the optimal iso-intensity value.

Inter-modality reproducibility: Estimated for manual measurements and the two segmentation methods as limits of agreement between the corresponding values on the results from the two modalities.

As the evaluation is based on limits of agreement, variability index and region overlap scores, we summarize their calculation details.

a. Limits of Agreement ( $L o A)$. In order to analyze the LoA between each segmentation method and the gold standard in a repeated study (multiple sessions), a twoway analysis of variance (ANOVA) with a statistical linear mixed model was used ${ }^{25}$. To obtain the standard deviation of the method with respect to the ideal gold standard (having zero variability), the variability of the gold standard $\sigma_{\mathrm{gs}}$ was removed from the standard devia-

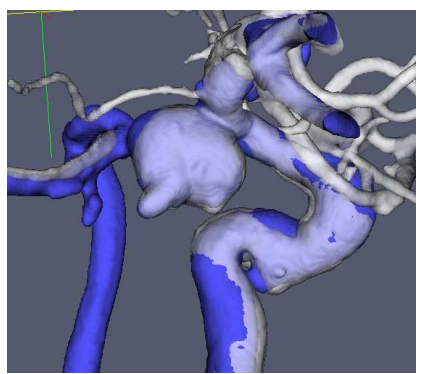

(a)

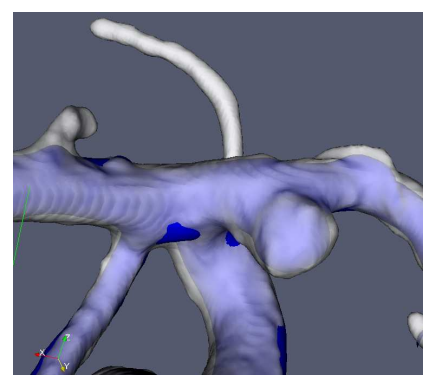

(b)
FIG. 4. Examples of the registered surfaces from the two modalities 3DRA (in white) and MRA (in blue). tion of $\operatorname{LoA}\left(\sigma_{\text {loa }}\right)$.

$$
\sigma_{\widehat{\mathrm{loa}}}=\sqrt{\sigma_{\mathrm{loa}}^{2}-\sigma_{\mathrm{gs}}^{2}}
$$

b. Variability index. To compare the deviation of segmentation results from the gold standard, with the variability of manual measurements, we defined the variability index (similar to the Williams index ${ }^{26}$ ) as the ratio of the method's standard deviation and the inter-observer variability of the manual measurements:

$$
I=\frac{\sigma_{\widehat{\text { oa }}}}{\sigma_{\text {inter-clinician }}}
$$

If the value of this index $I<1$, the segmentation method deviates from the gold standard less than the manual measurements vary between themselves.

c. Region overlap scores. The Dice and the conformity scores are defined as follows:

$$
\begin{gathered}
\text { Dice }=2 \frac{\left|S_{\text {method }} \cap S_{\mathrm{gs}}\right|}{\left|S_{\text {method }}\right|+\left|S_{\mathrm{gs}}\right|} \times 100 \% ; \\
\text { Conformity }=\left(1-\frac{\left|S_{\mathrm{FP}}\right|+\left|S_{\mathrm{FN}}\right|}{\left|S_{\mathrm{TP}}\right|}\right) \times 100 \%,
\end{gathered}
$$

where $S_{\text {method }}$ and $S_{\mathrm{gs}}$ are the sets of vessel region voxels labeled by the segmentation method and the gold standard, respectively. $S_{\mathrm{FP}}, S_{\mathrm{FN}}$ and $S_{\mathrm{TP}}$ are the sets of false positives, false negatives and true positive voxels, respectively, with respect to the gold standard. $|X|$ denotes the cardinality of the set $X$. Both scores reach the value of $100 \%$ in the case of perfect overlap and are mutually related by

$$
\text { Conformity }=3-\frac{2}{\text { Dice }}
$$




\section{RESULTS}

Results are grouped according to the region of interest (aneurysms or vessels) and according to the imaging modality (3DRA or MRA). The two widths used as descriptors of vessel and aneurysm cross-sections were evaluated together. For reporting statistical significance, two-tailed $95 \%$ confidence intervals were considered.

\section{A. Qualitative Analysis}

The following effects were causing erroneous segmentations in certain cutting planes:

Touching vessel effect: Two vessels are merged and the $1^{\text {st }}$ width is the sum of both vessel diameters. It occurred mostly in 3DRA images where small vessels can appear very close or touching. In TOFMRA images, due to the low spatial resolution and the low blood flow in small vessels, these vessels are usually not visible in the images.

Missing vessel effect: The cutting plane contains no segmentation contour. It happened mostly in TOFMRA images due to the low signal in a vessel.

Wide aneurysm neck: The aneurysm dome is merged with the surrounding vasculature. Hence, the neck is largely oversegmented.

Indented aneurysm: Aneurysm neck and dome become severely undersegmented (shape is deformed) due to the low signal in the image. It happened only in TOF-MRA images, where the low signal is caused by flow induced artifacts (e.g. turbulent flow).

Examples of the above-mentioned effects are shown in Fig. 5. The percentage of occurrence for each specific modality and segmentation method is shown in Table I.

The occurrence rate of touching vessels in GAR was half of the value in ISE for 3DRA images (close to being statistically significant, $p=0.06$, binomial-test) and significantly smaller than in ISE for MRA images $(p=0.01$, binomial-test). The missing vessel effect was rare for both segmentation methods. In contrast, the problems at the aneurysm were equally frequent for both GAR and ISE $(p>0.8$, binomial-test). The aneurysm neck is especially susceptible to qualitative errors since in many cases it is hard to approximate a neck using a $2 \mathrm{D}$ plane.

\section{B. Quantitative Analysis}

\section{Repeatability}

The variability of the GAR segmentation results is mainly due to the training set and depends on the number of images used to produce it. This variability is

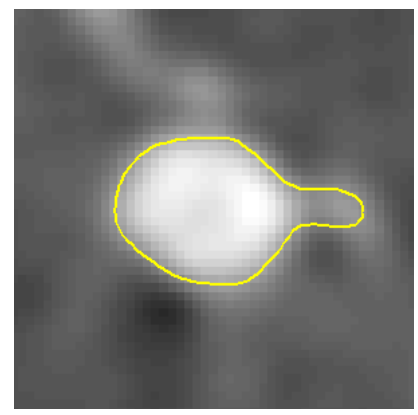

(a)

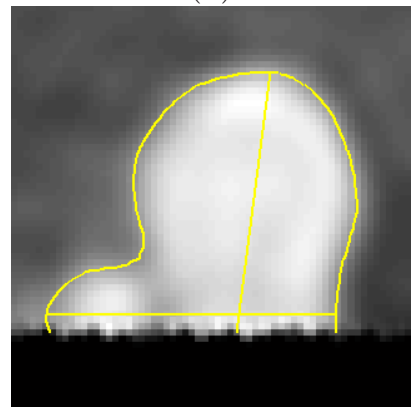

(c)

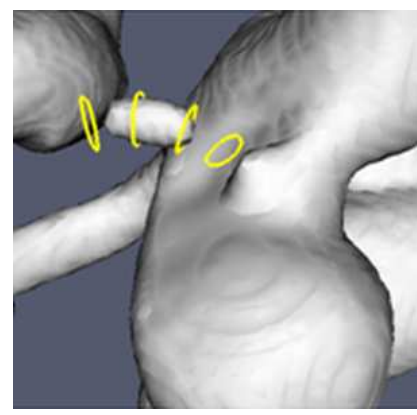

(b)

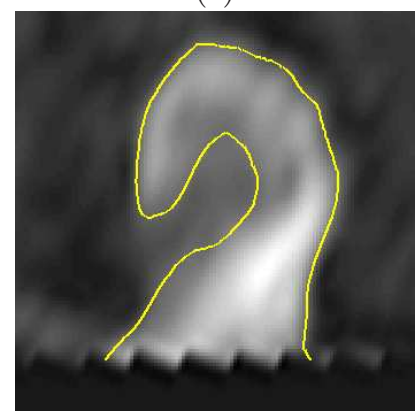

(d)
FIG. 5. Examples of qualitative errors: Touching vessel effect (a), missing vessel effect (b), wide aneurysm neck (c) and indented aneurysm (d).

expected to decrease as the size of the training set increases, because the variability introduced by manually segmenting each image is canceled out when averaging more images. To evaluate the repeatability of GAR, the segmentation of the 10 3DRA images was repeated with 16 different training sets: 4 sizes were selected to build the training set (number of images: 3, 6, 8 and 10) and, for each size, 4 independent (non-overlapping) sets of images were used. For training sets having 10 images, the inter-training set variability was less than $0.05 \mathrm{~mm}$ for the vessel and aneurysm regions (Fig. 6).

The operator-induced variability values for manual measurements and ISE segmentation method are presented in Table II. The variability of the GAR method was clearly lower than even the intra-observer variability of the ISE and the manual measurements. The rest of the presented GAR results on 3DRA correspond to those made with 4 different training sets composed from 10 images each.

\section{Accuracy}

Limits of agreement of GAR and ISE with the gold standard are presented in Table III and displayed in Fig. 7(a) where they are compared to the inter-observer variability of manual measurements. The error is shown in Fig. 7(b) and is expressed as the average difference (in absolute values) between the segmentation methods and the gold standard. The variability index (Eq. 4) is shown 
TABLE I. Percentage of excluded cutting planes due to the occurrence of qualitative segmentation errors. Per modality there were: 300 planes positioned on vessels (10 patients, 30 planes each), and 30 planes positioned on aneurysms (10 patients, 1 plane positioned on the neck and 2 on the dome).

\begin{tabular}{|c|c|c|c|c|}
\hline \multirow{3}{*}{ Effect } & \multicolumn{4}{|c|}{ Occurrence rate [\%] } \\
\hline & \multicolumn{2}{|c|}{ GAR } & \multicolumn{2}{|c|}{ ISE } \\
\hline & 3DRA & MRA & 3DRA & MRA \\
\hline Touching vessel effect & $8.7(26 / 300)$ & $0.3(1 / 300)$ & $16(48 / 300)$ & $5.7(17 / 300)$ \\
\hline Missing vessel effect & $0(0 / 300)$ & $2(6 / 300)$ & $0.3(1 / 300)$ & $2(6 / 300)$ \\
\hline Wide aneurysm neck & $40(4 / 10)$ & $20(2 / 10)$ & $30(3 / 10)$ & $30(3 / 10)$ \\
\hline Indented aneurysm & $0(0 / 30)$ & $17(5 / 30)$ & $0(0 / 30)$ & $10(3 / 30)$ \\
\hline
\end{tabular}

TABLE II. Standard deviations (SD) of variability (two-way ANOVA) with their 95\% confidence interval (CI), for manual measurements and ISE segmentation method.

\begin{tabular}{|c|c|c|c|c|c|}
\hline & \multirow{3}{*}{ Method } & \multicolumn{4}{|c|}{$S D \pm 95 \%$ CI $[\mathrm{mm}]$} \\
\hline & & \multicolumn{2}{|c|}{ Vessel } & \multicolumn{2}{|c|}{ Aneurysm } \\
\hline & & 3DRA & MRA & 3DRA & MRA \\
\hline \multirow{2}{*}{ Inter-observer } & Manual & $0.27 \pm 0.02$ & $0.30 \pm 0.02$ & $0.30 \pm 0.05$ & $0.29 \pm 0.05$ \\
\hline & ISE & $0.18 \pm 0.01$ & $0.22 \pm 0.01$ & $0.15 \pm 0.03$ & $0.24 \pm 0.05$ \\
\hline \multirow{2}{*}{ Intra-observer } & Manual & $0.17 \pm 0.01$ & $0.18 \pm 0.01$ & $0.26 \pm 0.05$ & $0.20 \pm 0.04$ \\
\hline & ISE & $0.14 \pm 0.01$ & $0.16 \pm 0.01$ & $0.11 \pm 0.02$ & $0.20 \pm 0.04$ \\
\hline
\end{tabular}

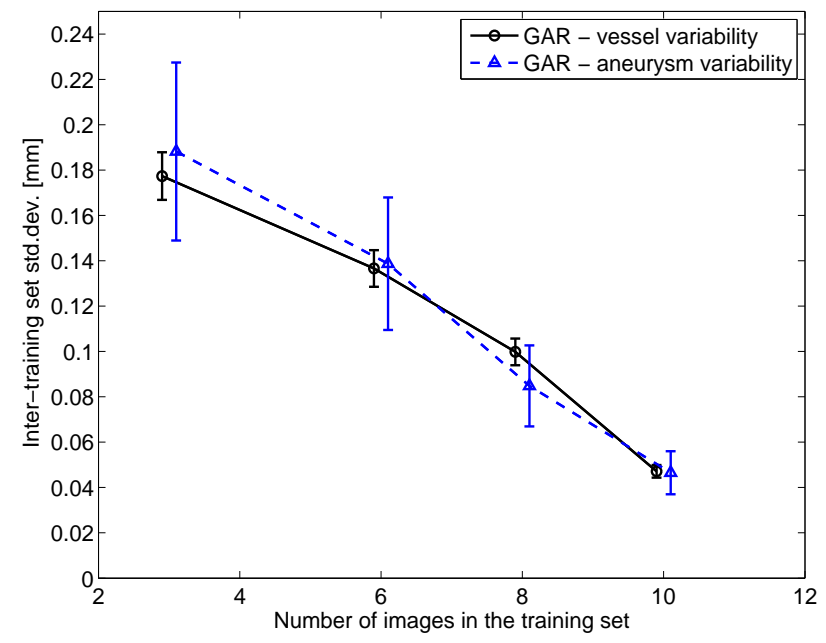

FIG. 6. Inter-training set variability (from 4 different training sets) of GAR in 3DRA in relation to the number of images $(3,6,8$ and 10) used to build the training sets. Error bars present the $95 \%$ confidence intervals.

\section{in Fig. 7(c).}

With the exception of the vessel region in MRA, the following observations are valid for GAR. The $95 \%$ limits of agreement were within those of manual measurements. The bias, although statistically significant, was small: less than one third of the image spatial resolution $(\sim 0.3 \mathrm{~mm}$ for $3 \mathrm{DRA}$ and $\sim 0.5 \mathrm{~mm}$ for MRA) and two to three times smaller than the standard deviation. The average error, when equally weighted between vessel and aneurysm region, was $0.2 \mathrm{~mm}$ for 3DRA and 0.27 $\mathrm{mm}$ for TOF-MRA, which was similar to that of manual measurements. The variability index was below 1 . Compared to ISE, GAR performed similarly in the ves- sel region and clearly better in the aneurysm region. In vessel region for MRA, both ISE and GAR performed worse than the manual measurements.

\section{Region overlap}

Manual voxel labeling has been performed by two operators on 3DRA and MRA images of the first 4 patients in a region of interest positioned around aneurysm, having an average size of $340000(70 \times 70 \times 70)$ voxels. The region overlap scores between each of the two segmentation methods and the manual segmentations are shown in Fig. 8, and are compared to the corresponding score between both manual segmentations as a measure of the inter-observer variability. The scores were averaged from all pairs of images and gold standard manual segmentations.

The overlap scores with the gold standard, for both segmentation methods were larger or comparable to the inter-observer ones. Thus, no notable mis-segmentation occurred for neither of the methods. Overlap scores for GAR and ISE were similar which suggests that most of the differences between the results of the two methods are at the sub-voxel level.

\section{Efficiency}

The GAR execution time depends on the size of the evolving surface i.e. the amount of vasculature being segmented. On average, for a cuboidal region of interest with a size of $256^{3}$ voxels, the execution time was $17 \pm$ 4 min (standard deviation) on a standard $\mathrm{PC}$ with an Intel quad-core $2.4 \mathrm{GHz}$ processor and $4 \mathrm{~GB}$ of memory. 
TABLE III. Agreement between the segmentation methods and the gold standard, expressed as bias with its $95 \%$ confidence interval (CI) and the population's standard deviation (SD) (computed with Eq. 3).

\begin{tabular}{|c|c|c|c|c|}
\hline \multirow{3}{*}{ Method } & \multicolumn{4}{|c|}{ LoA with gold standard: bias $\pm 95 \%$ CI (SD) [mm] } \\
\hline & \multicolumn{2}{|c|}{ Vessel } & \multicolumn{2}{|c|}{ Aneurysm } \\
\hline & 3DRA & MRA & 3DRA & MRA \\
\hline GAR & $-0.09 \pm 0.03(0.27)$ & $-0.12 \pm 0.04(0.37)$ & $0.09 \pm 0.11(0.19)$ & $-0.14 \pm 0.10(0.22)$ \\
\hline ISE & $0.01 \pm 0.03(0.29)$ & $0.15 \pm 0.04(0.39)$ & $0.20 \pm 0.12(0.28)$ & $0.11 \pm 0.12(0.31)$ \\
\hline
\end{tabular}

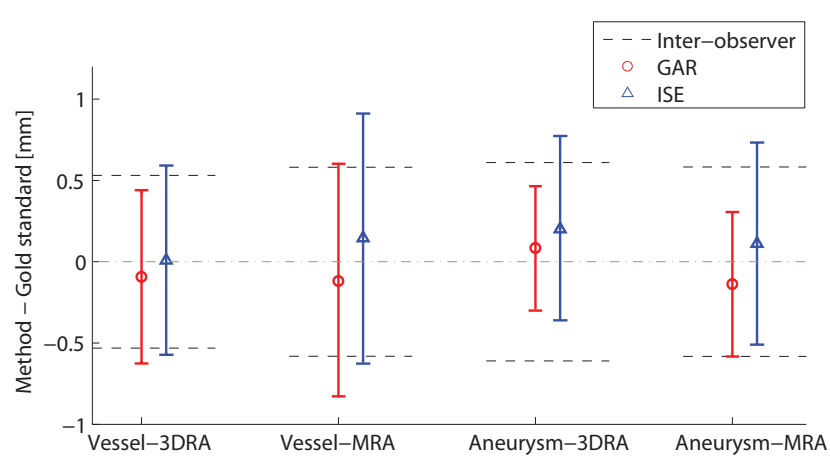

(a)

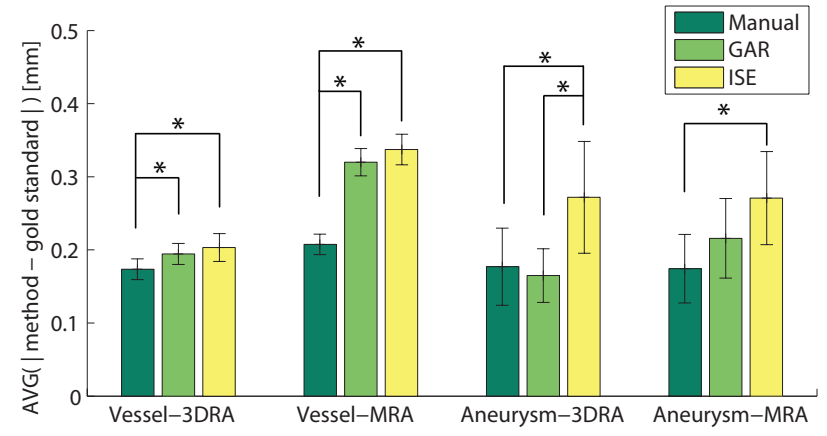

(b)

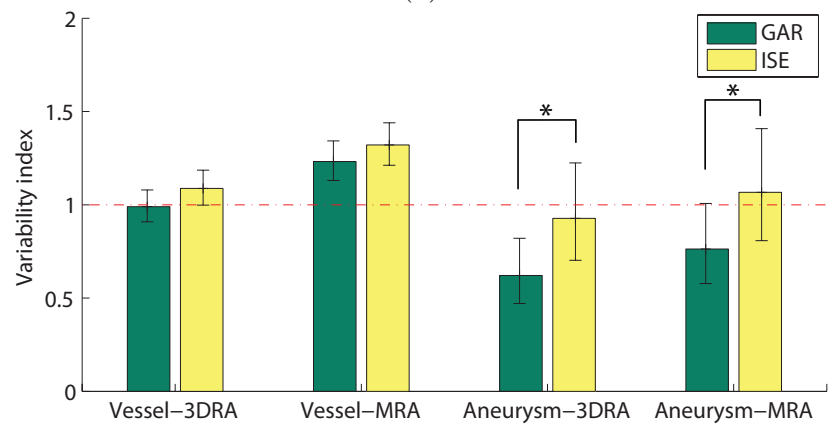

(c)

FIG. 7. (a) Limits of agreement between the segmentation method and the gold standard. Bias is denoted with a marker while the bars correspond to $95 \%$ limits of agreement $\left( \pm 2 \sigma_{\widehat{\text { loa }}}\right)$. Agreements are compared with inter-observer limits of agreement for the manual measurements. (b) Average absolute difference from the gold standard with 95\% confidence intervals. (c) Variability index with corresponding $95 \%$ confidence intervals. In (b) and (c), statistically significant difference $(p<0.05$, t-test $)$ is denoted with an asterisk $\left(^{*}\right)$.

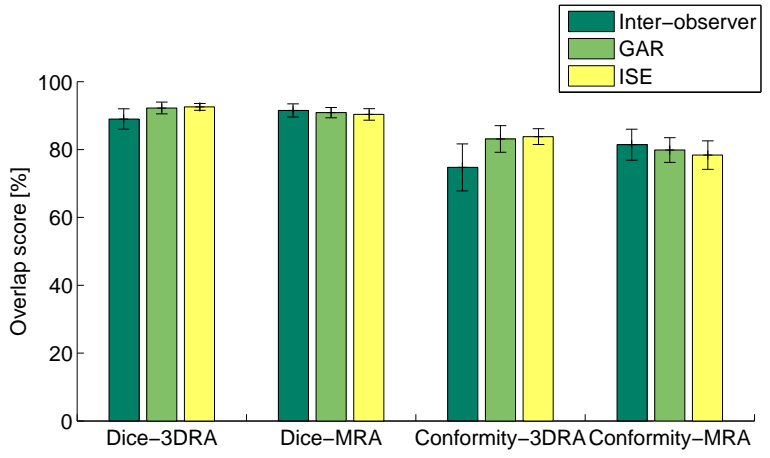

FIG. 8. Dice and conformity region overlap scores for the segmentation methods and the gold standard, with their $95 \%$ confidence intervals.

For ISE, on average $\sim 5$ min were required to decide on the optimal iso-intensity (the computational time for extraction of the iso-intensity surface was negligible).

\section{Inter-modality reproducibility}

Inter-modality agreement of manual measurements, and GAR and ISE methods are shown in Table IV. In the vessel region, GAR performed similar to manual measurements and both had a statistically significant bias. In the aneurysm region, the segmentation methods had large limits of agreement but were within those of the manual measurements. The variability introduced by the mesh registration procedure was $0.12 \pm 0.05 \mathrm{~mm}$ (standard deviation with its $95 \%$ confidence interval), which was small compared to the standard deviations of the inter-modality limits of agreement.

TABLE IV. Inter-modality limits of agreement, expressed as bias (MRA-3DRA) with its 95\% confidence interval (CI) and the population's standard deviation (SD).

\begin{tabular}{ccc}
\hline \hline \multirow{2}{*}{ Method } & \multicolumn{2}{c}{ Inter-modality LoA: bias \pm 95\% CI (SD) $[\mathrm{mm}]$} \\
\cline { 2 - 3 } & Vessel & Aneurysm \\
\hline Manual & $-0.21 \pm 0.04(0.48)$ & $0.03 \pm 0.22(0.84)$ \\
\hline GAR & $-0.24 \pm 0.04(0.49)$ & $-0.37 \pm 0.24(0.69)$ \\
\hline ISE & $-0.10 \pm 0.05(0.58)$ & $-0.17 \pm 0.25(0.75)$ \\
\hline \hline
\end{tabular}




\section{DISCUSSION}

We evaluated the GAR method's potential for segmenting cerebral vessels and aneurysms in 3DRA and MRA images acquired at different medical centers and with different imaging equipment. Three main improvements to the GAR method were introduced to make it suitable for segmenting images from clinical routine. Using an MVN classifier speeded up the region-based probability map computation. Using a voxel based feature scale selection enabled handling a larger variety of image spatial resolutions. The IIS component enabled the training set to be built irrespective of the clinical center and the imaging equipment.

As revealed by the qualitative analysis, the success of the segmentation depended on the local complexity of the vascular geometry, especially in the aneurysm region. Vessels which are very close to each other or to the aneurysm were sometimes merged in the extracted geometrical mesh. This partly happened because of insufficient imaging resolution, which means that more difficult geometrical configurations would require higher resolution and contrast to noise ratio for ensuring successful segmentation. In TOF-MRA images, segmentation was further hampered by large signal variations due to slow or turbulent flow.

In general, when qualitative segmentation errors occur, further interactive post-processing is required to remove the artifacts from the mesh. Favorably, the artifacts are of local nature and are easily detected visually. The methods used for their removal depend on the application. For the use in computational blood flow simulations, a sequence of local operations like mesh element removal and hole filling followed by an additional volume-preserving smoothing are typically applied to improve the mesh quality and to correct for geometrical and topological irregularities. There are various open-source tools already available, like $\mathrm{ReMESH}^{27}, \mathrm{MeshLab}^{28}$ or GIMIAS $^{29}$, which are able to efficiently repair triangular meshes. Repairing the mesh is time consuming and depends on the extent of the artifacts and the operator's experience. As a reference, a single touching vessel artifact can be removed in less than 5 min. However, limiting the occurrence of such artifacts is still a strong incentive for the research community to continue designing new segmentation algorithms.

The GAR method has been compared to the ISE method. The aim was to do the comparison with a method that is already clinically available. ISE is commonly used as a segmentation method on the commercial workstations of the imaging equipment, since the highest intensities in 3DRA and MRA images correspond to vessels $^{30}$.

The automatic GAR and interactive ISE methods differed from the gold standard within acceptable limits compared to the imaging resolution and had similar region overlap scores. GAR had an average accuracy of 0.2 $\mathrm{mm}$ for 3DRA and $0.27 \mathrm{~mm}$ for TOF-MRA, and had a repeatability of $0.05 \mathrm{~mm}$. Compared to ISE, GAR had a lower qualitative error in the vessel region and a lower quantitative error in the aneurysm region. Moreover, GAR is automatic and its repeatability was superior to both ISE and manual measurements. In contrast, the inter-observer variability of ISE could even result in differences in the extracted vascular topology, which would also mean adding a large variability to any subsequent vascular analysis.

Comparison of our evaluation setup and results to that of the current state of the art methods is given in Table V. All the methods use some variant of the deformable model and produce results with sub-voxel precision. Most require interactive initialization in the form of a seeded region growing. Manniesing et al. ${ }^{10}$ study is the most similar to ours. They also presented a fully automatic method capable of extracting the whole vascular tree, as opposed to other groups focusing on the interactive segmentation of individual vascular segments, and used manual measurements of the cross-sections as gold standard. However, they describe the shape with only one diameter, no observer variability was reported and no aneurysms were included in the evaluation. Chang et al. ${ }^{21}$ and Firouzian et al. ${ }^{22}$ used manual voxel-based segmentations as gold standard, loosing sub-voxel precision in the process. Firouzian et al. limited the evaluation to just the aneurysms but unlike Chang et al. they did report the inter-observer variability. Castro el $\mathrm{al}^{31}$. and Antiga et al. ${ }^{8}$ used digital and physical phantoms, respectively, which enabled them to estimate surface to surface distance to the gold standard. Castro et al. and Chang et al. combined the performance on vessels and aneurysms, which made the results biased towards the ones on vessels since aneurysms composed only a small part of the resulting volume or mesh. Finally, only Chang et al. compared their results with another method: intensity thresholding, a voxel-wise variant of ISE.

Ideally, the results of segmenting the same vasculature from two different modalities should coincide, although some differences might appear due to different image formation mechanisms. Of the two results from the two modalities, we assume that the one from 3DRA is closer to ground truth due to 3DRA's higher imaging resolution and contrast to noise ratio, which is also in agreement with the inter-observer variability being larger in MRA than in 3DRA. Thus, observing the inter-modality agreement in the vessel region, the GAR and the manual measurements tended to undersegment TOF-MRA on average. Probably, the actual vessel lumen was underestimated because of the very slow flow near the vessel wall and the saturation effects so the vessel seemed narrower in the cross-section image. In the aneurysm region we noticed differences in the shape of reconstructed aneurysms between 3DRA and MRA, especially for medium and large sized ones. This may be attributed to the difference in the contrast distribution, visible in 3DRA images, and the blood flow, visible in TOF-MRA images. Thus, although it was recently shown that $3 \mathrm{~T}$ TOF-MRA and 
CE-MRA were equivalent in evaluating the occlusion status of intracranial aneurysms ${ }^{32}$, CE-MRA might produce better images for the purpose of accurate aneurysm segmentation. However, understanding the cause of these differences between the two modalities goes beyond the scope of this study.

This evaluation study had some limitations. The most important one is that the performance was evaluated only on certain cutting plane positions, not on the entire extracted surface. In addition, cross-sections were only described with two measured widths. This decision was made to have less but meaningful measurements in a larger amount of images and processed by more clinicians. Otherwise the amount of required manual effort would have reduced the number of analyzed patients. However, we did perform a smaller study using region overlap scores and no notable mis-segmentations were observed. Lastly, the imprecision in establishing intermodality correspondences between the cutting planes might have influenced the inter-modality limits of agreement.

Taking all the evaluation results into account, the GAR performed better than ISE in accuracy and repeatability, also achieving a good agreement with the gold standard in 3DRA and in TOF-MRA imaging modalities. Thus, we conclude that GAR is able to segment 3DRA and TOF-MRA images coming from clinical routine and is better suited than ISE for extracting vascular geometry for use in a computational modeling process.

Automatic and repeatable image segmentation technique like GAR is essential to achieve reproducibility and consistency of the subsequent analysis steps in computational modeling pipelines. Such pipelines rely on the availability of accurate patient-specific vascular models and are able to streamline the creation of personalized anatomical, structural and haemodynamic models ${ }^{5}$. These are then used to derive robust and reliable quantitative descriptors providing an integrated decision support system to assess the risk of aneurysm rupture in patients and to optimize their treatments.

\section{Appendix: Multiscale feature vector}

The region-based information of GAR is represented in the form of a probability map associated with a particular region $R$. For the application of vessel segmentation in 3DRA and MRA we define two regions: vessel region and background region. The estimated probability value at each voxel $x$ of the probability map represents the conditional probability, $P(x \in R \mid \mathbf{f}(x))$, that voxel $x$ belongs to region $R$ observing the feature vector $\mathbf{f}(x)$. The feature vector $\mathbf{f}(x)$ is built from differential invariants ${ }^{33}$ up to the second order and in the multiscale framework ${ }^{34}$. Differential invariants are invariant to rigid transformations but not to scale, thus they are computed at several scales (having standard deviations: $\sigma_{0} \ldots \sigma_{m}$ ).

$$
\mathbf{f}(\mathbf{x})=\left(f_{\sigma_{0}}, \ldots, f_{\sigma_{m}}\right)(\mathbf{x})
$$

The set of derivatives used at one scale consists of the local jet of order two $\left(L, L_{i}, L_{j}^{i}\right)$ from which we compute the invariants. The formulation is given in Eq. A.2, using Einstein tensor notation:

$\mathbf{f}_{\sigma_{n}}(\mathbf{x})=\left(L, L_{i}^{i}, L_{i} L^{i}, L_{j}^{i} L_{i}^{j}, L_{i} L_{j}^{i} L^{j}, L_{j}^{i} L_{k}^{j} L_{i}^{k}, L_{i} L_{j}^{i} L_{k}^{j} L^{k}\right)$.

When calculating the local jet, the image is convolved with a Gaussian kernel having standard deviation $\sigma_{n}$, which is related to the scale at which we compute the local-jet. Given the impossibility of computing the invariants at all scales, we need to quantize the scales.

To deal with the large variety of possible image resolutions, the scales used for feature calculations are based on voxel spacing and not on world spacing. It assumes that small vessels look like large ones just on a different scale so the number of voxels per vessel diameter is the important factor ${ }^{35}$ instead of the vessel width in $\mathrm{mm}$. By modeling the intrinsic image resolution as a Gaussian point spread function with standard deviation of half a voxel size, the standard deviation of the applied Gaussian filter kernel is taken as:

$$
\sigma_{n}=\sqrt{\lambda_{n}^{2}-0.5^{2}}
$$

where the scales in voxel units are:

$$
\lambda_{n}=0.5 \exp (n \delta)
$$

The scale sampling parameter $\delta$ was chosen to be 0.3 and the number of scales as $5, n=0, \ldots, 4$. Both present a compromise between the density of sampling in the scale space and the computational requirements. As a result, the standard deviations of Gaussian filter kernels used are $\sigma_{n}=\{0,0.5535,0.9941,1.5830,2.4255\}$, expressed in voxel units. Having 7 features per scale, this produces for each voxel a 35 dimensional feature vector.

\section{ACKNOWLEDGMENTS}

The authors would like to acknowledge J. Schneiders for providing part of the TOF-MRA imaging data. The authors would like to thank also A.G. Radaelli, M. Nieber and X. Planes for the help in implementing some of the methods. This work was partially supported by the @neurIST Integrated Project (co-financed by the European Commission through the contract no. IST-027703), the CDTI CENIT-CDTEAM grant funded by the Spanish Ministry of Science and Innovation (MICINN-CDTI), the AGAUR-FI fellowship from Generalitat de Catalunya and Philips Healthcare (Best, The Netherlands).

\footnotetext{
${ }^{1}$ J.L. Brisman, J.K. Song, and D.W. Newell, "Cerebral aneurysms," N. Engl. J. Med. 355, 928-939 (Aug. 2006).
} 
${ }^{2}$ A.M. McKinney, C.S. Palmer, C.L. Truwit, A. Karagulle, and M. Teksam, "Detection of aneurysms by 64 -section multidetector CT angiography in patients acutely suspected of having an intracranial aneurysm and comparison with digital subtraction and 3D rotational angiography," AJNR Am. J. Neuroradiol. 29 594-602 (Mar. 2008).

${ }^{3}$ W.J. van Rooij, M.E. Sprengers, A.N. de Gast, J.P.P. Peluso, and M. Sluzewski, "3D rotational angiography: the new gold standard in the detection of additional intracranial aneurysms," AJNR Am. J. Neuroradiol. 29, 976-979 (May 2008).

${ }^{4}$ Y. Hiratsuka, H. Miki, I. Kiriyama, K. Kikuchi, S. Takahashi, I. Matsubara, K. Sadamoto, and T. Mochizuki, "Diagnosis of unruptured intracranial aneurysms: 3T MR angiography versus 64-channel multi-detector row CT angiography," Magn. Reson. Med. Sci. 7, 169-178 (2008).

${ }^{5}$ M.C. Villa-Uriol, I. Larrabide, J.M. Pozo, M. Kim, O. Camara, M. De Craene, C. Zhang, A.J. Geers, H. Morales, H. Bogunovic, R. Cardenes, and A.F. Frangi, "Toward integrated management of cerebral aneurysms," Philos. Trans. R. Soc. A-Math. Phys. Eng. Sci. 368, 2961-2982 (Jun. 2010).

${ }^{6}$ M.L. Raghavan, B. Ma, and R.E. Harbaugh, "Quantified aneurysm shape and rupture risk," J. Neurosurg. 102, 355-362 (Feb. 2005).

${ }^{7}$ R.D. Millan, L. Dempere-Marco, J.M. Pozo, J.R. Cebral, and A.F. Frangi, "Morphological characterization of intracranial aneurysms using 3-D moment invariants," IEEE Trans. Med. Imag. 26, 1270-1282 (Sep. 2007).

${ }^{8}$ L. Antiga, M. Piccinelli, L. Botti, B. Ene-Iordache, A. Remuzzi, and D.A. Steinman, "An image-based modeling framework for patient-specific computational hemodynamics," Med. Biol. Eng. Comput. 46, 1097-1112 (Nov. 2008).

${ }^{9}$ J.R. Cebral, M.A. Castro, S. Appanaboyina, C.M. Putman, D. Millan, and A.F. Frangi, "Efficient pipeline for image-based patient-specific analysis of cerebral aneurysm hemodynamics: technique and sensitivity," IEEE Trans. Med. Imag. 24, 457-467 (Apr. 2005).

${ }^{10}$ R. Manniesing, M.A. Viergever, A. van der Lugt, and W.J. Niessen, "Cerebral arteries: Fully automated segmentation from CT angiography - a feasibility study," Radiology 247, 841-846 (2008).

${ }^{11}$ M. Hernandez and A.F. Frangi, "Non-parametric geodesic active regions: method and evaluation for cerebral aneurysms segmentation in 3DRA and CTA," Med. Image Anal. 11, 224-241 (Jun. 2007).

${ }^{12}$ H. Bogunovic, A.G. Radaelli, M. De Craene, D. Delgado, and A.F. Frangi, "Image intensity standardization in 3D rotational angiography and its application to vascular segmentation," in Proc. SPIE Med. Imag., Vol. 6914, edited by J. M. Reinhardt and J. P. W. Pluim (2008) article 691419.

${ }^{13}$ S.J. Osher and R.P. Fedkiw, Level Set Methods and Dynamic Implicit Surfaces (Springer-Verlag, 2002).

${ }^{14}$ I. Aizenberg, N. Aizenberg, and J. Vandewalle, Multi-Valued and Universal Binary Neurons: Theory, Learning, Applications (Kluwer Academic, 2000).

${ }^{15}$ L.G. Nyul and J.K. Udupa, "On standardizing the MR image intensity scale," Magn. Reson. Med. 42, 1072-1081 (Dec. 1999).

${ }^{16}$ R.H. Byrd, P. Lu, J. Nocedal, and C. Zhu, "A limited memory algorithm for bound constrained optimization," SIAM J. Sci. Comput. 16, 1190-1208 (1995), ISSN 1064-8275.

${ }^{17}$ D. Rueckert, P. Aljabar, R.A. Heckemann, J.V. Hajnal, and A. Hammers, "Diffeomorphic registration using B-splines," in Proc. Int. Conf. Med. Imag. Comput. \& Comput. Assist. Interven. (MICCAI), Lect. Notes Comput. Sci., Vol. 4191, edited by R. Larsen, M. Nielsen, and J. Sporring (2006) pp. 702-709.

${ }^{18}$ W.E. Lorensen and H.E. Cline, "Marching cubes: A high resolution 3D surface construction algorithm," Comput. Graph. (ACM) 21, 163-169 (Jul. 1987).
${ }^{19}$ P.J. Besl and H.D. McKay, "A method for registration of 3-D shapes," IEEE Trans. Pattern Anal. Mach. Intell. 14, 239-256 (1992).

${ }^{20}$ J.K. Udupa, V.R. Leblanc, Y. Zhuge, C. Imielinska, H. Schmidt, L.M. Currie, B.E. Hirsch, and J.Woodburn, "A framework for evaluating image segmentation algorithms," Comp. Med. Imag. Graph. 30, 75-87 (Mar. 2006).

${ }^{21}$ H.-H. Chang, G.R. Duckwiler, D.J. Valentino, and W.C. Chu, "Computer-assisted extraction of intracranial aneurysms on 3D rotational angiograms for computational fluid dynamics modeling," Med. Phys. 36, 5612-5621 (2009).

${ }^{22}$ A. Firouzian, R. Manniesing, H.Z. Flach, R. Risselada, F. van Kooten, M.C. Sturkenboom, A. van der Lugt, and W.J. Niessen, "Intracranial aneurysm segmentation in 3D CT angiography: Method and quantitative validation with and without prior noise filtering," Eur. J. Radiol.(2010), article in Press.

${ }^{23}$ L.R. Dice, "Measures of the amount of ecologic association between species," Ecology 26, 297-302 (Jul. 1945).

${ }^{24}$ H-H. Chang, A.H. Zhuang, D.J. Valentino, and W-C. Chu, "Performance measure characterization for evaluating neuroimage segmentation algorithms," NeuroImage 47, 122-35 (Aug. 2009).

${ }^{25}$ B. Carstensen, J. Simpson, and L.C. Gurrin, "Statistical models for assessing agreement in method comparison studies with replicate measurements," Int. J. Biostat. 4 (2008), article 16.

${ }^{26}$ V. Chalana and Y. Kim, "A methodology for evaluation of boundary detection algorithms on medical images," IEEE Trans. Med. Imag. 16, 642-652 (Oct. 1997).

${ }^{27} \mathrm{M}$. Attene and B. Falcidieno, "ReMESH: an interactive environment to edit and repair triangle meshes," in Proc. IEEE Int. Conf. Shape Model. Appl. (SMI) (2006) pp. 271-276.

28 "MeshLab: an open source, portable, and extensible system for the processing and editing of unstructured 3D triangular meshes," http://meshlab.sourceforge.net (2010), [Online; accessed 2010-09-30].

${ }^{29}$ I. Larrabide, P. Omedas, Y. Martelli, X. Planes, M. Nieber, J.A. Moya, C. Butakoff, R. Sebastian, O. Camara, M. De Craene, B.H. Bijnens, and A.F. Frangi, "GIMIAS: an open source framework for efficient development of research tools and clinical prototypes," in Proc. Int. Conf. Funct. Imag. Model. Heart. (FIMH), Lect. Notes Comput. Sci., Vol. 5528, edited by N. Ayache, H. Delingette, and M. Sermesant (2009) pp. 417-426.

${ }^{30}$ M. Piotin, P. Gailloud, L. Bidaut, S. Mandai, M. Muster, J. Moret, and D.A. Rufenacht, "CT angiography, MR angiography and rotational digital subtraction angiography for volumetric assessment of intracranial aneurysms. An experimental study," Neuroradiology 45, 404-409 (Jun. 2003).

${ }^{31}$ M.A. Castro, C.M. Putman, and J.R. Cebral, "Patient-specific computational modeling of cerebral aneurysms with multiple avenues of flow from 3D rotational angiography images," Acad. Radiol. 13, 811-821 (Jul. 2006).

${ }^{32}$ M.E.S. Sprengers, J.D. Schaafsma, W.J. van Rooij, R. van den Berg, G.J.E. Rinkel, E.M. Akkerman, S.P. Ferns, and C.B.L.M. Majoie, "Evaluation of the occlusion status of coiled intracranial aneurysms with MR angiography at 3T: Is contrast enhancement necessary?." AJNR Am. J. Neuroradiol. 30, 1665-1671 (Jul. 2009).

${ }^{33}$ L.M.J. Florack, B.M. ter Haar Romeny, J.J. Koenderink, and M.A. Viergever, "General intensity transformations and differential invariants," J. Math. Imag. Vis. 4, 171-187 (May 1994).

${ }^{34}$ C. Schmid and R. Mohr, "Local grayvalue invariants for image retrieval," IEEE Trans. Pattern Anal. Mach. Intell. 19, 530-535 (May 1997).

${ }^{35}$ R.M. Hoogeveen, C.J. Bakker, and M.A. Viergever, "Limits to the accuracy of vessel diameter measurement in MR angiography," J. Magn. Reson. Imag. 8, 1228-1235 (1998). 


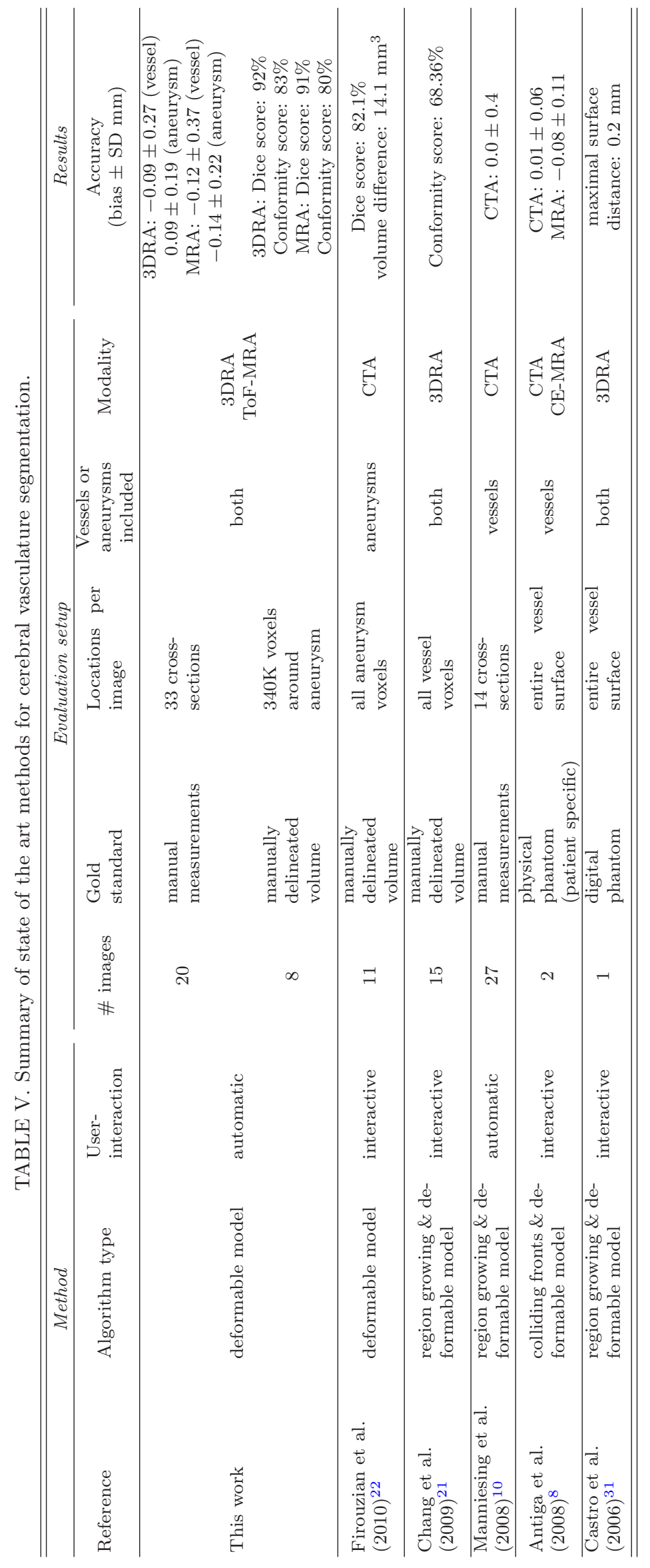

